Research Article

\title{
Vertex identification in trees
}

\author{
Yuya Kono, Ping Zhang* \\ Department of Mathematics, Western Michigan University, Kalamazoo, Michigan 49008-5248, USA
}

(Received: 23 April 2021. Accepted: 13 July 2021. Published online: 16 July 2021.)

(c) 2021 the authors. This is an open access article under the CC BY (International 4.0) license (www.creativecommons.org/licenses/by/4.0/).

\begin{abstract}
A red-white coloring of a nontrivial connected graph $G$ of diameter $d$ is an assignment of red and white colors to the vertices of $G$ where at least one vertex is colored red. Associated with each vertex $v$ of $G$ is a $d$-vector, called the code of $v$, whose $i$ th coordinate is the number of red vertices at distance $i$ from $v$. A red-white coloring of $G$ for which distinct vertices have distinct codes is called an identification coloring or ID-coloring of $G$. A graph $G$ possessing an ID-coloring is an ID-graph. The minimum number of red vertices among all ID-colorings of an ID-graph $G$ is the identification number or ID-number of $G$. Necessary conditions are established for those trees that are ID-graphs. A tree $T$ is starlike if $T$ is obtained by subdividing the edges of a star of order 4 or more. It is shown that for every positive integer $r$ different from 2 , there exist starlike trees satisfying some prescribed properties having ID-number $r$.
\end{abstract}

Keywords: distance; vertex identification; identification coloring; tree.

2020 Mathematics Subject Classification: 05C05, 05C12, 05C15, 05 C90.

\section{Introduction}

Over the years, many methods have been introduced with the goal of uniquely identifying the vertices of a connected graph. Often these approaches have employed distance and coloring. The oldest of these methods deal with what is referred to as the metric dimension of a connected graph. For a nontrivial connected graph $G$ of order $n$, the goal is to locate an ordered set $W=\left\{w_{1}, w_{2}, \ldots, w_{k}\right\}$ of $k$ vertices in $G, 1 \leq k \leq n$, and associate with each vertex $v$ of $G$ the $k$-vector $\left(a_{1}, a_{2}, \ldots, a_{k}\right)$, where $a_{i}$ is the distance $d\left(v, w_{i}\right)$ between $v$ and $w_{i}, 1 \leq i \leq k$. If the $n k$-vectors produced in this manners are distinct, then the vertices of $G$ have been uniquely identified. For each connected graph $G$, such a set $W$ can always be found since we can always choose $W=V(G)$. The primary problem here is to determine the minimum size of such a set $W$. This is referred to as the metric dimension of $G$. Equivalently, the metric dimension of a connected graph $G$ can be defined as the minimum number of vertices of $G$ that can be assigned the same color, say red, such that for every two vertices $u$ and $v$ of $G$, there exists a red vertex $w$ such that $d(u, w) \neq d(v, w)$. This parameter is defined for every connected graph.

Another method that has been studied to uniquely identify the vertices of a connected graph $G$ has been referred to as the partition dimension of $G$. For a nontrivial connected graph $G$ of order $n$, the goal is to obtain a $k$-coloring, $1 \leq k \leq n$, of the vertices of $G$, where the coloring is not required (or expected) to be a proper coloring. This results in $k$ color classes $V_{1}, V_{2}, \ldots, V_{k}$ of $V(G)$. For each vertex $v$ of $G$, we once again associate a vector, here a $k$-vector $\left(a_{1}, a_{2}, \ldots, a_{k}\right)$ where $a_{i}$ denotes the distance from $v$ to a nearest vertex in $V_{i}$ for $1 \leq i \leq k$. If the vertices of $G$ have distinct $k$-vectors, then the vertices of $G$ have been uniquely identified. Such a coloring always exists since we can always assign distinct colors to the vertices of $G$, thereby obtaining a procedure that has similarity to metric dimension. The minimum number of colors that accomplishes this goal is referred to as the partition dimension of $G$. The partition dimension of a connected graph $G$ can also be defined as the minimum number $k$ of colors (denoted by $1,2, \ldots, k$ ) that can be assigned to the vertices of $G$, one color to each vertex, so that for every two vertices $u$ and $v$ of $G$, there exists a color $i$ such that the distance between $u$ and a nearest vertex colored $i$ is distinct from the distance between $v$ and a nearest vertex colored $i$. This parameter is also defined for every connected graph.

Another method that has been introduced for the purpose of uniquely identifying the vertices of a connected graph is referred to as an identification coloring. Let $G$ be a connected graph of diameter $d \geq 2$ and let there be given a redwhite vertex coloring $c$ of the graph $G$ where at least one vertex is colored red. That is, the color $c(v)$ of a vertex $v$ in $G$ is either red or white and $c(v)$ is red for at least one vertex $v$ of $G$. With each vertex $v$ of $G$, there is associated a $d$-vector $\vec{d}(v)=\left(a_{1}, a_{2}, \ldots, a_{d}\right)$ called the code of $v$ corresponding to $c$, where the $i$ th coordinate $a_{i}$ is the number of red vertices at

\footnotetext{
*Corresponding author (ping.zhang@wmich.edu).
} 
distance $i$ from $v$ for $1 \leq i \leq d$. If distinct vertices of $G$ have distinct codes, then $c$ is called an identification coloring or ID-coloring. Equivalently, an identification coloring of a connected graph $G$ is an assignment of the color red to a nonempty subset of $V(G)$ (with the color white assigned to the remaining vertices of $G$ ) such that for every two vertices $u$ and $v$ of $G$, there is an integer $k$ with $1 \leq k \leq d$ such that the number of red vertices at distance $k$ from $u$ is different from the number of red vertices at distance $k$ from $v$. A graph possessing an identification coloring is an ID-graph. A major difference here from the two methods described above is that not all connected graphs are ID-graphs.

The concept of metric dimension was introduced independently by Slater [15] and by Harary and Melter [10] and has been studied by many (see [4,7], for example). Slater described the usefulness of these ideas when working with U.S. Coast Guard Loran (long range aids to navigation) stations in [15, 16]. Johnson [13,14] of the former Upjohn Pharmaceutical Company applied this in attempts to develop the capability of large datasets of chemical graphs. The concept of partition dimension was introduced in [6]. These concepts as well as other methods of vertex identifications in graphs have been studied by many with various applications (see $[2,3,8,9,11,12,17,18]$ for example). The concepts of ID-colorings and ID-graphs were introduced and studied in [5].

All of the methods mentioned above involve constructing a vertex coloring of a connected graph $G$ with the goal of producing a vertex labeling of $G$ (using vectors of the same size as labels) so that distinct vertices of $G$ have the distinct labels. Consequently, the goal of each of these methods is to obtain an irregular labeling of $G$. The general topic of irregularity in graphs is described in [2]. There is the related topic of obtaining a labeling of $G$ by means of colorings where exactly two vertices of $G$ have the same label. These are called artiregular labelings, a topic discussed in [1].

We first present five results obtained in [5] on ID-colorings. For an integer $t \geq 2$, the members of a set $S$ of $t$ vertices of a graph $G$ are called $t$-tuplets (twins if $t=2$ and triplets if $t=3$ ) if either (1) $S$ is an independent set in $G$ and every two vertices in $S$ have the same neighborhood or (2) $S$ is a clique, that is the subgraph $G[S]$ induced by $S$ is complete and every two vertices in $S$ have the same closed neighborhood.

Proposition 1.1. Let $c$ be an ID-coloring of a connected graph $G$. If $u$ and $v$ are twins of $G$, then $c(u) \neq c(v)$. Consequently, if $G$ is an ID-graph, then $G$ is triplet-free.

Proposition 1.2. Let $c$ be a red-white coloring of a connected graph $G$ where there is at least one vertex of each color. If $x$ is a red vertex and $y$ is a white vertex, then $\vec{d}(x) \neq \vec{d}(y)$.

Theorem 1.1. A nontrivial connected graph $G$ has $\operatorname{ID}(G)=1$ if and only if $G$ is a path.

Theorem 1.2. A connected graph $G$ of diameter 2 is an ID-graph if and only if $G=P_{3}$.

Theorem 1.3. For a positive integer $r$, there exists a connected graph $G$ with $\operatorname{ID}(G)=r$ if and only if $r \neq 2$.

The following result describes a property of ID-colorings.

Proposition 1.3. Let $G$ be a connected graph with an ID-coloring c. If $H$ is a connected subgraph of $G$ such that $(i) H$ contains all red vertices in $G$ and $(i i) d_{G}(x, y)=d_{H}(x, y)$ for every two vertices $x$ and $y$ of $H$, then the restriction of $c$ to $H$ is an ID-coloring of $H$.

Proof. Let $\operatorname{diam}(H)=d$ and let $c_{H}$ be the restriction of $c$ to $H$. For a vertex $v$ of $H$, let $\vec{d}_{c_{H}}(v)=\left(a_{1}^{\prime}, a_{2}^{\prime}, \ldots, a_{d}^{\prime}\right)$ and let $\vec{d}_{c}(v)=\left(a_{1}, a_{2}, \ldots, a_{d}, \ldots\right)$. Notice that if $d=\operatorname{diam}(G)$, then $\vec{d}_{c}(v)=\left(a_{1}, a_{2}, \ldots, a_{d}\right)$ while if $d<\operatorname{diam}(G)$, then $a_{t}=0$ for each integer $t$ with $d+1 \leq t \leq \operatorname{diam}(G)$. Since $H$ contains all red vertices in $G$ and $d_{G}(v, w)=d_{H}(v, w)$ for every vertex $w$ of $G$, it follows that $a_{i}=a_{i}^{\prime}$ for $1 \leq i \leq d$ and so the restriction of $c$ to $H$ is an ID-coloring of $H$.

Both conditions stated in the hypothesis of Proposition 1.3 for a connected subgraph $H$ of a graph $G$ are needed. For example, consider the ID-graph $G$ in Figure 1. The subgraph $H_{1}$ of $G$ does not contain all red vertices of $G$ while the subgraph $H_{2}$ is not distance-preserving. For $i=1,2$, the restriction of the ID-coloring $c$ of $G$ to the subgraph $H_{i}$ of $G$ is not an ID-coloring of $H_{i}$ (since there are twins both of which are colored white).

Here, our emphasis turns to trees that are ID-graphs, namely ID-trees. We investigate structural problems of ID-trees, provide necessary conditions for trees to be ID-trees, and establish a realization result on ID-numbers of ID-trees satisfying some prescribed conditions.

\section{ID-colorings of trees}

The only $t$-tuplets, $t \geq 2$, in a tree $T$ are end-vertices of $T$, all with the same neighbor. As we saw, if $T$ contains triplets, then $T$ is not an ID-tree. If $T$ contains twins and possesses an ID-coloring, then the twins must be colored differently in every ID-coloring. We now see that for trees, the concepts of twins and triplets are special cases of something more general. 


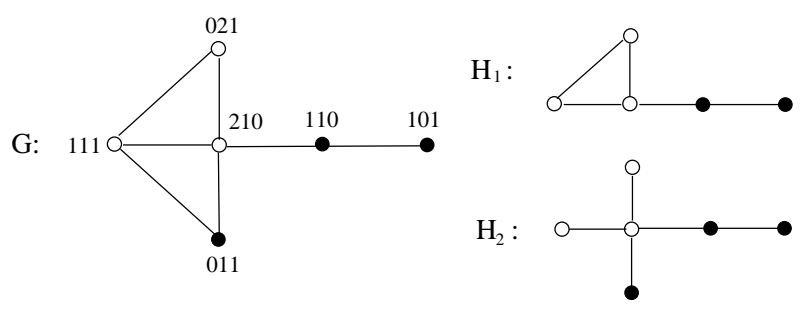

Figure 1: Two subgraphs of an ID-graph $G$.

If $T$ is a tree with a vertex $v$ possessing two isomorphic branches $B_{1}$ and $B_{2}$, then $B_{1}$ and $B_{2}$ are twin branches at $v$ if there is an isomorphism from $B_{1}$ to $B_{2}$ fixing $v$. If $T$ contains a vertex $v$ possessing three isomorphic branches $B_{1}, B_{2}$, and $B_{3}$ such that every two of them are twin branches, then $B_{1}, B_{2}$, and $B_{3}$ are triplet branches at $v$. If the size of each branch at $v$ is 1 , then $T$ contains twins or triplets. For example, there are three isomorphic branches of size 5 at the vertex $v$ of the tree $T$ of Figure 2. However, $T$ has twin branches at $v$ but no triplet branches at $v$.



Figure 2: A tree $T$ with twin branches of size 5 at $v$.

Let $T_{1}$ and $T_{2}$ be two rooted trees whose roots are $v_{1}$ and $v_{2}$, respectively. Then $T_{1}$ and $T_{2}$ are considered to be isomorphic rooted trees, denoted $T_{1} \cong T_{2}$, if there is an isomorphism $\alpha: V\left(T_{1}\right) \rightarrow V\left(T_{2}\right)$ such that $\alpha\left(v_{1}\right)=v_{2}$. For $i=1,2$, let $c_{i}$ be a red-white coloring of a tree $T_{i}$ rooted at $v_{i}$ where $T_{1} \cong T_{2}$. Then $c_{1}$ and $c_{2}$ are considered to be isomorphic colorings, denoted $c_{1} \cong c_{2}$, if there is an isomorphism $\alpha: V\left(T_{1}\right) \rightarrow V\left(T_{2}\right)$ such that $\alpha\left(v_{1}\right)=v_{2}$ and $c_{1}(x)=c_{2}(\alpha(x))$ for every vertex $x$ of $T_{1}$. In particular, $c_{1}\left(v_{1}\right)=c_{2}\left(v_{2}\right)$.

Observation 2.1. Suppose that a tree $T$ has twin branches $B_{1}$ and $B_{2}$ at a vertex $v$ and $c$ is a red-white coloring of $T$. For $i=1,2$, let $c_{i}$ be the restriction of $c$ to $B_{i}$ rooted at $v$. If $c_{1} \cong c_{2}$, then $c$ is not an ID-coloring of $T$.

Let $T_{0}$ be a tree of size $k \geq 1$ rooted at a vertex $v$. If the color of $v$ is fixed, say $v$ is white, then there are at most $2^{k}$ distinct (non-isomorphic) red-white colorings of $T_{0}$ in which $v$ is colored white. Consequently, if there are more than $2^{k}$ copies of a particular branch of size $k$ at $v$, then $T$ is not an ID-tree by Observation 2.1. In the case when $k=1$, this simply says that no ID-tree can contain a triplet.

Let $T$ be a tree rooted at a vertex $v$ and let $c$ be an ID-coloring of $T$. If $T_{0}$ is a subtree of $T$ of minimum order rooted at $v$ such that $T_{0}$ contains all red vertices in $T$, then the restriction of $c$ to $T_{0}$ is an ID-coloring of $T_{0}$ by Proposition 1.3. Necessarily, all end-vertices of $T_{0}$ are red. In the case when $T_{0}$ is a path $P_{k+1}$ of size $k$ whose end-vertices are $v$ and $w$, there are at most $2^{k-1}$ distinct red-white colorings of $P_{k+1}$ in which $v$ is white and $w$ is red.

A tree $T$ is starlike if $T$ is obtained by subdividing the edges of a star of order 4 or more. Equivalently, a tree $T$ is starlike if and only if $T$ has exactly one vertex whose degree is greater than 2 . This vertex is referred to as the central vertex of $T$. If the degree of the central vertex $v$ of a starlike $T$ is $k \geq 3$, then $T$ has $k$ branches (paths) at $v$, each branch containing $v$ as an end-vertex of $T$. For example, the starlike tree $T$ in Figure 3 has four branches at its central vertex. This tree is twin-free but does contain twin branches at its central vertex. This starlike tree is an ID-graph and an ID-coloring having exactly four red vertices is shown in Figure 3. In fact, $\operatorname{ID}(T)=4$.

Proposition 2.1. Let $T$ be a starlike tree whose largest branch at its central vertex $v$ has size $k$. If $T$ is an ID-tree, then for each integer $i$ with $1 \leq i \leq k$, there are at most $2^{i}$ branches of size $i$ or less at $v$. Consequently, if $T$ is an ID-tree, then $T$ has at most $2^{k}$ branches at $v$.

Proof. In view of Proposition 1.3, it suffices to determine the maximum number of distinct red-white colorings of all branches (paths) of $T$ such that $v$ is white and all end-vertices are red. For each integer $i$ with $1 \leq i \leq k$, there are $2^{i-1}$ distinct red-white colorings of branches of size $i$ at $v$ in which $v$ is colored white and the other end-vertex of each 


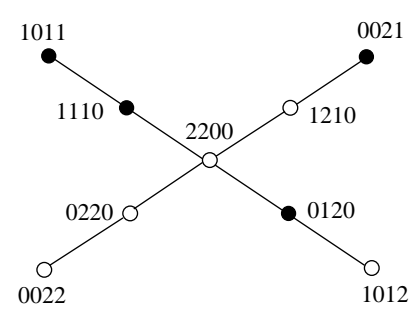

Figure 3: An ID-coloring of a twin-free starlike tree.

branch is colored red. Thus, the minimum number of branches of size $i$ at $v$ without duplicating a red-white coloring of these branches is $2^{i-1}$. Therefore, the maximum number of all such red-white colorings of branches of all possibles sizes at $v$ is $\sum_{i=1}^{k} 2^{i-1}=2^{k}-1$. Since there can be one branch of size $k$ or less at $v$ all of whose vertices are colored white, it follows that there can be $2^{k}$ branches at $v$ such that the red-white colorings of every two isomorphic branches at $v$ are different.

Corollary 2.1. Let $T$ be a starlike tree whose largest branch at its central vertex $v$ has size $k$. If $T$ has more than $2^{k}$ branches at $v$, then $T$ is not an ID-tree.

For example, if $T$ is a starlike ID-tree whose largest branch at its central vertex $v$ has size 3 , then (1) there are at most two branches of size 1 at $v,(2)$ there are at most four branches of size 2 or less at $v$, and (3) there are at most eight branches of size 3 or less at $v$. As an illustration, the three starlike trees of Figure 4 satisfy all conditions (1)-(3).

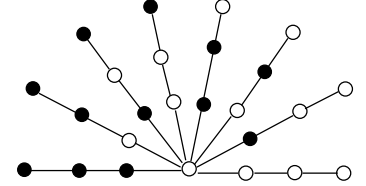

(a)

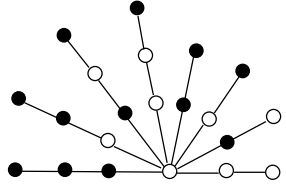

(b)

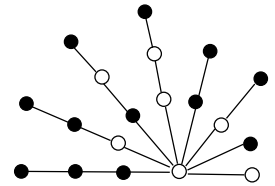

(c)

Figure 4: Three starlike trees whose largest branch at its central vertex has size 3.

The tree of Figure 4(a) has eight branches of size 3 at its central vertex and no branches of size less than 3 at its central vertex. The tree of Figure 4(b) has four branches of size 3, four branches of size 2, and no branches of size 1 at its central vertex. The tree of Figure 4(c) has four branches of size 3, two branches of size 2, and two branches of size 1 at its central vertex. In each case, there are eight branches at the central vertex of the tree. The red-white colorings of the three trees in Figure 4 are essentially the same coloring. It can be shown that this coloring is an ID-coloring. For the red-white coloring of the tree $T$ of Figure 4(c), partial codes of the vertices of $T$ containing the initial coordinates of each code are shown in Figure 5. (These partial codes are sufficient to show that all codes are distinct.) Since every two distinct vertices of $T$ have distinct codes, this red-white coloring is an ID-coloring of $T$.

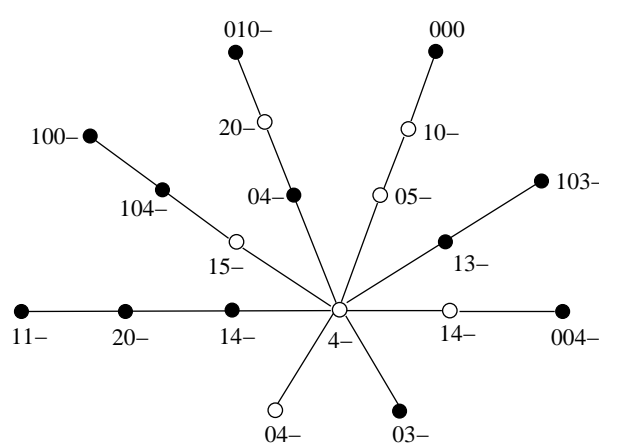

Figure 5: An ID-coloring of a starlike tree.

Theorem 2.1. If $T$ is a starlike tree with central vertex $v$ whose branches at $v$ have distinct sizes, then $T$ is an ID-tree.

Proof. Suppose that $\operatorname{deg} v=k \geq 3$ and let $B_{1}, B_{2}, \ldots, B_{k}$ be the branches (paths) of $T$ at $v$, where $B_{i}$ has size $m_{i}$ and $m_{i}<m_{i+1}$ for $1 \leq i<k$. Define a red-white coloring $c$ of $T$ that assigns the color white to $v$ and the color red to all other vertices of $T$. We show that $c$ is an ID-coloring of $T$. By Proposition 1.2, it suffices to show that every two red vertices have 
distinct codes. Let $x, y \in V(T)-\{v\}$ and let $\vec{d}(x)=\left(a_{1}, a_{2}, \ldots, a_{d}\right)$ and $\vec{d}(y)=\left(b_{1}, b_{1}, \ldots, b_{d}\right)$ where $d=\operatorname{diam}(T)=m_{k-1}+m_{k}$. Suppose that $d(x, v)=s$ and $d(y, v)=t$. We consider two cases, according to whether $s \neq t$ or $s=t$.

Case 1. $s \neq t$, say $s<t$. Then $a_{s} \in\{0,1\}$ and $b_{s} \in\{1,2\}$. If $a_{s} \neq b_{s}$, then $\vec{d}(x) \neq \vec{d}(y)$. Thus, we may assume that $a_{s}=b_{s}=1$. Thus, $a_{s+1} \in\{k-1, k\}$ and $b_{s+1} \in\{0,1\}$. Since $k \geq 3$, it follows that $a_{s+1} \geq 2$ and so $a_{s+1} \neq b_{s+1}$, implying that $\vec{d}(x) \neq \vec{d}(y)$.

Case 2. $s=t$. Then $x$ and $y$ belong to different branches of $T$ at $v$, say $x \in V\left(B_{i}\right)$ and $y \in V\left(B_{j}\right)$ where $1 \leq i<j \leq k$. Let $B_{i}=\left(v=v_{0}, v_{1}, \ldots, v_{m_{i}}\right)$ and $B_{j}=\left(v=u_{0}, u_{1}, \ldots, u_{m_{j}}\right)$, where then $x=v_{s}$ and $y=u_{s}$. If $m_{i}-s+1=s$, then $a_{m_{i}-s+1}=0$ and $b_{m_{i}-s+1} \geq 1$. If $m_{i}-s+1 \neq s$, then $b_{m_{i}-s+1}=a_{m_{i}-s+1}+1$. In either case, $\vec{d}(x) \neq \vec{d}(y)$. Therefore, $c$ is an ID-coloring of $T$.

\section{Starlike trees with prescribed ID-number}

We saw in Theorem 1.3 that for every integer $r \geq 3$, there exists a connected graph $G$ with $\operatorname{ID}(G)=r$. For such a given integer $r$, the graph $G$ described in the proof of Theorem 1.3 contains $r$ pairwise disjoint twins from which it follows that $\operatorname{ID}(G) \geq r$. It was therefore only necessary to show that $\operatorname{ID}(G) \leq r$. We now show that for every integer $r \geq 3$, there exists a tree $T$ with no twins at all such that $\operatorname{ID}(T)=r$. In addition, we show that there is a tree without twin branches having IDnumber $r$. In particular, we show that for every odd integer $r \geq 5$ there is a twin-free tree $T$ whose automorphism group contains $(r+1)$ ! elements such that $\operatorname{ID}(T)=r$. We also show that there is a red-white coloring $c$ of the same class of trees $T$ where exactly $r-1$ vertices are colored red such that $\vec{d}(x)=\vec{d}(y)$ for exactly one pair $x, y$ of vertices of $T$. Consequently, there is a red-white coloring of these trees $T$ with exactly two vertices having the same code. As we metioned earlier, such a (red-white) coloring results in an antiregular labeling (see [1,2], for example.)

For each integer $r \geq 3$, let $T=S_{r-1}\left(K_{1, r+1}\right)$ be the starlike tree obtained from the star $K_{1, r+1}$ of order $r+2$ by subdividing each edge of the $r+1$ edges in $K_{r+1}$ exactly $r-1$ times. Let $v$ be the central vertex of $T$. Then the degree of $v$ is $r+1$ and each of the $r+1$ branches of $T$ at $v$ has length $r$. For each integer $i$ with $0 \leq i \leq r$, let $B_{i}=\left(v, v_{i, 1}, v_{i, 2}, \ldots, v_{i, r}\right)$ be a branch of $T$ at $v$. Then $\operatorname{diam}(T)=2 r$ and $T$ is twin-free.

Theorem 3.1. For each odd integer $r \geq 3, \operatorname{ID}\left(S_{r-1}\left(K_{1, r+1}\right)\right)=r$.

Proof. For an odd integer $r \geq 3$, let $T=S_{r-1}\left(K_{1, r+1}\right)$, where $v$ is the central vertex of $T$ and $B_{i}=\left(v, v_{i, 1}, v_{i, 2}, \ldots, v_{i, r}\right)$ is a branch of $T$ at $v$ for $0 \leq i \leq r$. First, we show that $\operatorname{ID}(T) \geq r$. For any red-white coloring of $T$ that assigns the color red to at most $r-1$ vertices of $T$, there are at least two branches, say $B_{0}$ and $B_{1}$, of $T$ at $v$ such that the paths $B_{0}-v$ and $B_{1}-v$ contain no red vertices of $T$. However then, $\vec{d}\left(v_{0,1}\right)=\vec{d}\left(v_{1,1}\right)$, for example, and so this red-white coloring is not an ID-coloring of $T$. Therefore, $\operatorname{ID}(T) \geq r$.

Next, we show that $T$ has an ID-coloring with exactly $r$ red vertices. Define a red-white coloring $c$ of $T$ by assigning the color red to each vertex $v_{i, i}$ for $1 \leq i \leq r$ and white to the remaining vertices of $T$. Thus, $T$ has exactly $r$ red vertices. It remains to show that $c$ is an ID-coloring of $T$. Since $\operatorname{diam}(T)=2 r$, the code of each vertex of $T$ is a $(2 r)$-vector. Let $x$ and $y$ be two distinct vertices of $T$. We consider two cases, according to whether $x$ and $y$ are both red or both white. Let $\vec{d}(x)=\left(a_{1}, a_{2}, \ldots, a_{2 r}\right)$ and $\vec{d}(y)=\left(b_{1}, b_{2}, \ldots, b_{2 r}\right)$.

Case 1. $x$ and $y$ are both red. Let $x=v_{i, i}$ and $y=v_{j, j}$ where $1 \leq i<j \leq r$.

$\star$ First, suppose that $j \neq r$. Since (1) the last nonzero coordinate in $\vec{d}\left(v_{i, i}\right)$ is the $(i+r)$ th coordinate where $i+r=$ $d\left(v_{i, i}, v_{r, r}\right)$ and the last nonzero coordinate in $\vec{d}\left(v_{j, j}\right)$ is the $(j+r)$ th coordinate where $j+r=d\left(v_{j, j}, v_{r, r}\right)$ and $(2) i<j$, it follows that $a_{j+r}=0$ and $b_{j+r}=1$ and so $\vec{d}(x) \neq \vec{d}(y)$.

$\star$ Next, suppose that $j=r$. We saw that the last nonzero coordinate in $\vec{d}\left(v_{i, i}\right)$ where $1 \leq i \leq r-1$ is the $(i+r)$ th coordinate. Since the last nonzero coordinate in $\vec{d}\left(v_{r, r}\right)$ is the $(2 r-1)$ th coordinate where $2 r-1=d\left(v_{r-1, r-1}, v_{r, r}\right)$, it follows that if $i \neq r-1$, then $\vec{d}(x) \neq \vec{d}(y)$. Thus, we may assume that $x=v_{r-1, r-1}$. Because the first nonzero coordinate in $\vec{d}\left(v_{r-1, r-1}\right)$ is the $r$ th coordinate where $r=d\left(v_{1,1}, v_{r-1, r-1}\right)$ and the first nonzero coordinate in $\vec{d}\left(v_{r, r}\right)$ is the $(r+1)$ th coordinate where $r+1=d\left(v_{1,1}, v_{r, r}\right)$, it follows that $a_{r}=1$ and $b_{r}=0$ and so $\vec{d}(x) \neq \vec{d}(y)$.

Case 2. $x$ and $y$ are both white. First, we make some observations on the codes of vertices on $B_{0}$.

- The vertices on $B_{0}$ are the only white vertices of $T$ whose codes contain the $r$-tuple $(1,1, \ldots, 1)=1^{r}$ as a subsequence. The vertex $v$ is the only white vertex of $T$ such that the first $r$ coordinates of its code are 1 (that is, $\vec{d}(v)=\left(1^{r}, 0^{r}\right)$ ). For $1 \leq t \leq r$, the vertex $v_{0, t}$ is the only white vertex such that in $\vec{d}\left(v_{0, t}\right)$ the first $t$ coordinates and the last $r-t$ coordinates are 0 while the remaining coordinates are 1 (that is, $\vec{d}\left(v_{0, t}\right)=\left(0^{t}, 1^{r}, 0^{r-t}\right)$ for $1 \leq t \leq r$ ). Thus, all codes of the vertices of $B_{0}$ are distinct and they are also distinct from the codes of those white vertices that are not in $B_{0}$. 
Hence, we may assume that neither $x$ nor $y$ belongs to $B_{0}$. Let $Q_{i}=B_{i}-v=\left(v_{i, 1}, v_{i, 2}, \ldots, v_{i, r}\right)$ be the subpath of $B_{i}$ for $1 \leq i \leq r$. We consider two subcases, according to the location of $x$ and $y$.

Subcase 2.1. $x, y \in V\left(Q_{i}\right)$ where $1 \leq i \leq r$. Let $x=v_{i, p}$ and $y=v_{i, q}$ where $1 \leq p<q \leq r$ and $p, q \neq i$.

$\star$ First, suppose that $i \neq r$. Since (1) the last nonzero coordinate in $\vec{d}\left(v_{i, p}\right)$ is the $(p+r)$ th coordinate where $p+r=$ $d\left(v_{i, p}, v_{r, r}\right)$ and the last nonzero coordinate in $\vec{d}\left(v_{i, q}\right)$ is the $(q+r)$ th coordinate where $q+r=d\left(v_{i, q}, v_{r, r}\right)$ and (2) $p<q$, it follows that $a_{q+r-1}=0$ and $b_{q+r-1}=1$ and so $\vec{d}(x) \neq \vec{d}(y)$.

$\star$ Next, suppose that $i=r$. Since (1) the last nonzero coordinate in $\vec{d}\left(v_{r, p}\right)$ is the $(p+r-1)$ th coordinate where $p+r-1=$ $d\left(v_{i, p}, v_{r-1, r-1}\right)$ and the last nonzero coordinate in $\vec{d}\left(v_{r, q}\right)$ is the $(q+r-1)$ th coordinate where $q+r-1=d\left(v_{i, q}, v_{r-1, r-1}\right)$ and (2) $p<q$, it follows that $a_{q+r-1}=0$ and $b_{q+r-1}=1$ and so $\vec{d}(x) \neq \vec{d}(y)$.

Subcase 2.2. $x \in V\left(Q_{i}\right)$ and $y \in V\left(Q_{j}\right)$ where $1 \leq i<j \leq r$. Let $x=v_{i, p}$ and $y=v_{j, q}$ where $1 \leq p, q \leq r, p \neq i$, and $q \neq j$. We consider two subcases, according to whether $p=q$ or $p \neq q$.

Subcase 2.2.1. $p=q$. Then $x=v_{i, p}$ and $y=v_{j, p}$ where $1 \leq i<j \leq r$ and $p \notin\{i, j\}$.

$\star$ First, suppose that $j+1 \leq p \leq r$. Since (1) the first nonzero coordinate in $\vec{d}\left(v_{i, p}\right)$ is the $(p-i)$ th coordinate where $p-i=d\left(v_{i, p}, v_{i, i}\right)$ and the first nonzero coordinate in $\vec{d}\left(v_{j, p}\right)$ is the $(p-j)$ th coordinate where $p-j=d\left(v_{j, p}, v_{j, j}\right)$ and (2) $i<j$, it follows that $a_{p-j}=0$ and $b_{p-j}=1$ and so $\vec{d}(x) \neq \vec{d}(y)$.

$\star$ Next suppose that $1 \leq p \leq i-1$. Let $c_{0}$ be the red-white coloring of $T$ obtained by recoloring $v_{i, i}$ and $v_{j, j}$ white and all other vertices of $T$ remain the same colors as in $c$. Since $d\left(v_{i, p}, w\right)=d\left(v_{j, p}, w\right)$ for every red vertex $w$ such that $w \notin\left\{v_{i, i}, v_{j, j}\right\}$, it follows that $\vec{d}_{c_{0}}(x)=\vec{d}_{c_{0}}(y)=\left(f_{1}, f_{2}, \ldots, f_{2 r}\right)$. Observe that $d\left(v_{i, p}, v_{i, i}\right)=i-p, d\left(v_{i, p}, v_{j, j}\right)=j+p$, $d\left(v_{j, p}, v_{i, i}\right)=i+p$, and $d\left(v_{j, p}, v_{j, j}\right)=j-p$. Since $i-p<\min \{i+p, j-p, j+p\}$, it follows that $a_{i-p}=f_{i-p}+1$ and $b_{i-p}=f_{i-p}$ and so $\vec{d}(x) \neq \vec{d}(y)$.

* Finally, suppose that $i+1 \leq p \leq j-1$. Let $c_{0}$ be the red-white coloring of $T$ obtained by recoloring $v_{i, i}$ and $v_{j, j}$ white and all other vertices of $T$ remain the same colors as in $c$. Since $d\left(v_{i, p}, w\right)=d\left(v_{j, p}, w\right)$ for every red vertex $w$ such that $w \notin\left\{v_{i, i}, v_{j, j}\right\}$, it follows that $\vec{d}_{c_{0}}(x)=\vec{d}_{c_{0}}(y)=\left(f_{1}, f_{2}, \ldots, f_{2 r}\right)$. Observe that $d\left(v_{i, p}, v_{i, i}\right)=p-i, d\left(v_{i, p}, v_{j, j}\right)=j+p$, $d\left(v_{j, p}, v_{i, i}\right)=i+p$, and $d\left(v_{j, p}, v_{j, j}\right)=j-p$. Since $j+p>\max \{i+p, j-p, i+p\}$, it follows that $a_{j+p}=f_{j+p}+1$ and $b_{j+p}=f_{j+p}$ and so $\vec{d}(x) \neq \vec{d}(y)$.

Subcase 2.2.2. $p \neq q$. First, suppose that $j \neq r$. Since (1) the last nonzero coordinate in $\vec{d}\left(v_{i, p}\right)$ is the $(p+r)$ th coordinate where $p+r=d\left(v_{i, p}, v_{r, r}\right)$ and the last nonzero coordinate in $\vec{d}\left(v_{j, q}\right)$ is the $(q+r)$ th coordinate where $q+r=d\left(v_{j, q}, v_{r, r}\right)$ and (2) $p \neq q$, it follows that either $a_{p+r} \neq b_{p+r}$ or $a_{q+r} \neq b_{q+r}$, implying that $\vec{d}(x) \neq \vec{d}(y)$.

For simplification, we now introduce notation where a code is expressed when no 0 coordinate is given after the final nonzero coordinate of a code. For example, if a code of a vertex is a 7-tuple $(1,0,2,1,0,0,0)$, we simply write this code as the 4-tuple $(1,0,2,1)$.

Next, suppose that $j=r$. Thus, $x=v_{i, p}$ where $1 \leq i \leq r-1$ and $p \neq i$ and $y=v_{r, q}$ where $1 \leq q \leq r-1$ and $p \neq q$. We consider two possibilities.

Subcase 2.2.2.1. $2 \leq i \leq r-1$. First, suppose that $p \geq i+1$. Then $\vec{d}(x)=\vec{d}\left(v_{i, p}\right)=\left(0^{i-p-1}, 1,0^{i}, 1^{i-1}, 0,1^{r-i}\right)$. If $\vec{d}(y)=\vec{d}\left(v_{r, q}\right)$ contains a coordinate 2 , then $\vec{d}(x) \neq \vec{d}(y)$. Thus, we may assume that $d\left(v_{r, q}, v_{r, r}\right)=r-q \neq q+t$ for $1 \leq t \leq r-1$ and so $\vec{d}(y)=\vec{d}\left(v_{r, q}\right)=\left(0^{r-q-1}, 1,0^{2 q-r}, 1^{r-1}\right)$. Since $1^{r-1}$ is a subsequence in $\vec{d}(y)$ and is not a subsequence of $\vec{d}(x)$, it follows that $\vec{d}(x) \neq \vec{d}(y)$.

Next, suppose that $1 \leq p \leq i-1$. If $i-p \neq p+\ell$ for some $\ell \in[r]-\{i\}$, then $\left(1^{i-1}, 0,1^{r-i}\right)$ is a subsequence of $\vec{d}(x)$ and so there is no 2 as a coordinate of $\vec{d}(x)$. If $\vec{d}(y)=\vec{d}\left(v_{r, q}\right)$ contains a coordinate 2 , then $\vec{d}(x) \neq \vec{d}(y)$ and so $\vec{d}(y)=\vec{d}\left(v_{r, q}\right)=$ $\left(0^{r-q-1}, 1,0^{2 q-r}, 1^{r-1}\right)$. Thus, $\vec{d}(x) \neq \vec{d}(y)$. Hence, we may assume that $i-p=p+\ell$ for some $\ell \in[r]-\{i\}$ and so $i=2 p+\ell$. This implies that there is exactly one coordinate 2 of $\vec{d}(x)$, namely $a_{i-p}=2$. If $\vec{d}(y)$ has no coordinate 2 , then $\vec{d}(x) \neq \vec{d}(y)$. Hence, we assume that $\vec{d}(y)$ has coordinate 2. This implies that $d\left(v_{r, q}, v_{r, r}\right)=r-q=q+t$ for some $t \in[r-1]$ and $b_{r-q}=2$ is the only coordinate 2 in $\vec{d}(y)$. Hence, $i-p=r-q$ or $r-i=q-p$ and so $q>p$. There are two possibilities here. If $i-p=r-q<p+1$, then the second nonzero coordinate in $\vec{d}(x)$ is $a_{p+1}$ while the the second nonzero coordinate in $\vec{d}(y)$ is $b_{q+1}$. If $i-p=r-q>p+1$, then the first nonzero coordinate in $\vec{d}(x)$ is $a_{p+1}$ while the the fist nonzero coordinate in $\vec{d}(y)$ is $b_{q+1}$. In either case, $\vec{d}(x) \neq \vec{d}(y)$. Therefore, $c$ is an ID-coloring and $\operatorname{so} \operatorname{ID}(T)=r$.

Subcase 2.2.2.2. $\quad i=1$. Then $\vec{d}(x)=\vec{d}\left(v_{1, p}\right)=\left(0^{p-2}, 1,0,0,1^{r-1}\right)$. If $\vec{d}(y)=\vec{d}\left(v_{r, q}\right)$ contains a coordinate 2, then $\vec{d}(x) \neq \vec{d}(y)$. Thus, we may assume that $d\left(v_{r, q}, v_{r, r}\right)=r-q \neq q+t$ for $1 \leq t \leq r-1$. Since $r-q<q+r-1$, it follows that $r-q \leq q$ or $q \geq r / 2$. Then $\vec{d}(y)=\vec{d}\left(v_{r, q}\right)=\left(0^{r-q-1}, 1,0^{2 q-r}, 1^{r-1}\right)$. Thus, $p-2=r-q-1$ (or $p+q=r+1$ ), $2=2 q-r$ (or $r=2 q-2$ is even) and so $p=q-1$ (or $q=p+1$ ). Since $r$ is odd, it follows that $\vec{d}(x) \neq \vec{d}(y)$. 
The following is a consequence of Theorem 3.1.

Corollary 3.1. For each odd integer $r \geq 3$, there exist a twin-free starlike tree $T$ such that $\operatorname{ID}(T)=r$.

In the statement of Theorem 3.1, the condition that $r \geq 3$ is an odd integer is only required in Subcase 2.2.2.2. In fact, if $r \geq 4$ is an even integer, then there are exactly two white vertices in the red-white coloring described in the proof of Theorem 3.1, namely $v_{1, p}$ and $v_{r, q}$ where $q=p+1$ and $p+q=r+1$, that have the same code. That is, this red-white coloring is antiregular. Therefore, we have the following.

Proposition 3.1. For each even integer $r \geq 4$, there is an antiregular red-white coloring of the starlike tree $S_{r-1}\left(K_{1, r+1}\right)$ having exactly $r$ red vertices.

By the technique used in the proof of Theorem 3.1, the following result can be verified.

Proposition 3.2. Let $r \geq 4$ be an even integer. If $T$ is the starlike tree obtained by subdividing exactly one edge of $S_{r-1}\left(K_{1, r+1}\right)$, then $\operatorname{ID}(T)=r$.

None of the trees appearing in the results just above have the identity automorphism group. We next describe a class of trees having the identity automorphism group, where each such tree is necessarily twin-free, which can be used to show that for every integer $r \geq 3$, there is a tree $T$ with the identity automorphism group such that $\operatorname{ID}(T)=r$.

Theorem 3.2. For each integer $r \geq 3$, there is a starlike tree T of order $1+\left(\begin{array}{c}r+2 \\ 2\end{array}\right)$ having the identity automorphism group such that $\operatorname{ID}(T)=r$.

Proof. For each integer $r \geq 3$, let $K_{1, r+1}$ be the star of order $r+2$ with central vertex $v$ that is adjacent to the $r+1$ end-vertices $v_{1}, v_{2}, \ldots, v_{r+1}$. Let $T$ be the starlike tree obtained from the star $K_{1, r+1}$ be subdividing the edge $v v_{i}$ of $K_{1, r+1}$ exactly $i-1$ times for $1 \leq i \leq r+1$. In particular, $v v_{1}$ is not subdivided and $v v_{r+1}$ is subdivided exactly $r$ times. Thus, $T$ is twin-free, the order of $T$ is $1+\left(\begin{array}{c}r+2 \\ 2\end{array}\right)$ and $\operatorname{diam}(T)=2 r+1$. Since no two vertices of $T$ are similar, it follows that $T$ has the identity automorphism group. For each integer $i$ with $1 \leq i \leq r+1$, let $B_{i}=\left(v, v_{i, 1}, v_{i, 2}, \ldots, v_{i, i}\right)$ be a branch of $T$ at $v$.

First, we show that $\operatorname{ID}(T) \geq r$. For any red-white coloring of $T$ that assigns the color red to at most $r-1$ vertices of $T$, there are at least two branches $B_{i}$ and $B_{j}$ of $T$ at $v$ such that the paths $B_{i}-v$ and $B_{j}-v$ contain no red vertices of $T$. However then, $\vec{d}\left(v_{i, 1}\right)=\vec{d}\left(v_{j, 1}\right)$, for example, and so this red-white coloring is not an ID-coloring of $T$. Therefore, $\operatorname{ID}(T) \geq r$. Next, we show that $T$ has an ID-coloring with exactly $r$ red vertices. Define a red-white coloring $c$ of $T$ by assigning the color red to each vertex $v_{i, i}$ for $1 \leq i \leq r$ and white to the remaining vertices of $T$. Thus, $T$ has exactly $r$ red vertices. It remains to show that $c$ is an ID-coloring of $T$. Since $\operatorname{diam}(T)=2 r+1$, the code of each vertex of $T$ is a $(2 r+1)$-vector. Let $x$ and $y$ be two distinct vertices of $T$. We consider two cases, according to whether $x$ and $y$ are both red or both white. Let $\vec{d}(x)=\left(a_{1}, a_{2}, \ldots, a_{2 r+1}\right)$ and $\vec{d}(y)=\left(b_{1}, b_{2}, \ldots, b_{2 r+1}\right)$.

Case 1. $x$ and $y$ are both red. Let $x=v_{i, i}$ and $y=v_{j, j}$ where $1 \leq i<j \leq r$.

$\star$ First, suppose that $j \neq r$. Since (1) the last nonzero coordinate in $\vec{d}\left(v_{i, i}\right)$ is the $(i+r)$ th coordinate where $i+r=$ $d\left(v_{i, i}, v_{r, r}\right)$ and the last nonzero coordinate in $\vec{d}\left(v_{j, j}\right)$ is the $(j+r)$ th coordinate where $j+r=d\left(v_{j, j}, v_{r, r}\right)$ and (2) $i<j$, it follows that $a_{j+r}=0$ and $b_{j+r}=1$ and so $\vec{d}(x) \neq \vec{d}(y)$.

* Next, suppose that $j=r$. We saw that the last nonzero coordinate in $\vec{d}\left(v_{i, i}\right)$ where $1 \leq i \leq r-1$ is the $(i+r)$ th coordinate. Since the last nonzero coordinate in $\vec{d}\left(v_{r, r}\right)$ is the $(2 r-1)$ th coordinate where $2 r-1=d\left(v_{r-1, r-1}, v_{r, r}\right)$, it follows that if $i \neq r-1$, then $\vec{d}(x) \neq \vec{d}(y)$. Thus, we may assume that $x=v_{r-1, r-1}$. Because the first nonzero coordinate in $\vec{d}\left(v_{r-1, r-1}\right)$ is the $r$ th coordinate where $r=d\left(v_{1,1}, v_{r-1, r-1}\right)$ and the first nonzero coordinate in $\vec{d}\left(v_{r, r}\right)$ is the $(r+1)$ th coordinate where $r+1=d\left(v_{1,1}, v_{r, r}\right)$, it follows that $a_{r}=1$ and $b_{r}=0$ and so $\vec{d}(x) \neq \vec{d}(y)$.

Case 2. $x$ and $y$ are both white. First, we verify the following claim.

Claim. If $x \in V\left(B_{r+1}\right)$ or $y \in V\left(B_{r+1}\right)$, then $\vec{d}(x) \neq \vec{d}(y)$.

The vertices on $B_{r+1}$ are the only white vertices of $T$ whose codes contain the $r$-tuple $(1,1, \ldots, 1)=1^{r}$ as a subsequence. The vertex $v$ is the only white vertex of $T$ such that the first $r$ coordinates of its code are 1 (that is, $\vec{d}(v)=\left(1^{r}, 0^{r+1}\right)$ ). For $1 \leq t \leq r+1$, the vertex $v_{r+1, t}$ is the only white vertex such that in $\vec{d}\left(v_{r+1, t}\right)$ the first $t$ coordinates and the last $r+1-t$ coordinates are 0 while the remaining coordinates are 1 (that is, $\vec{d}\left(v_{r+1, t}\right)=\left(0^{t}, 1^{r}, 0^{r+1-t}\right)$ for $1 \leq t \leq r$ ). Thus, all codes of the vertices of $B_{r+1}$ are distinct and they are also distinct from the codes of those white vertices that are not in $B_{r+1}$. Hence, the claim holds.

By the claim, we may assume that $x \notin V\left(B_{r+1}\right)$ and $y \notin V\left(B_{r+1}\right)$. Let $Q_{i}=B_{i}-v=\left(v_{i, 1}, v_{i, 2}, \ldots, v_{i, i}\right)$ be the subpath of $B_{i}$ for $2 \leq i \leq r$. We consider two subcases, according to the location of $x$ and $y$.

Subcase 2.1. $x, y \in V\left(Q_{i}\right)$ where $2 \leq i \leq r$. Let $x=v_{i, p}$ and $y=v_{i, q}$ where $1 \leq p<q \leq i-1$. 
$\star$ First, suppose that $i \neq r$. Since (1) the last nonzero coordinate in $\vec{d}\left(v_{i, p}\right)$ is the $(p+r)$ th coordinate where $p+r=$ $d\left(v_{i, p}, v_{r, r}\right)$ and the last nonzero coordinate in $\vec{d}\left(v_{i, q}\right)$ is the $(q+r)$ th coordinate where $q+r=d\left(v_{i, q}, v_{r, r}\right)$ and (2) $p<q$, it follows that $a_{q+r-1}=0$ and $b_{q+r-1}=1$ and so $\vec{d}(x) \neq \vec{d}(y)$.

$\star$ Next, suppose that $i=r$. Since (1) the last nonzero coordinate in $\vec{d}\left(v_{r, p}\right)$ is the $(p+r-1)$ th coordinate where $p+r-1=$ $d\left(v_{i, p}, v_{r-1, r-1}\right)$ and the last nonzero coordinate in $\vec{d}\left(v_{r, q}\right)$ is the $(q+r-1)$ th coordinate where $q+r-1=d\left(v_{i, q}, v_{r-1, r-1}\right)$ and (2) $p<q$, it follows that $a_{q+r-1}=0$ and $b_{q+r-1}=1$ and so $\vec{d}(x) \neq \vec{d}(y)$.

Subcase 2.2. $x \in V\left(Q_{i}\right)$ and $y \in V\left(Q_{j}\right)$ where $2 \leq i<j \leq r$. Let $x=v_{i, p}$ and $y=v_{j, q}$ where $1 \leq p \leq i-1$ and $1 \leq q \leq j-1$. We consider two subcases, according to whether $p=q$ or $p \neq q$.

Subcase 2.2.1. $p=q$. Then $x=v_{i, p}$ and $y=v_{j, p}$ where $2 \leq i<j \leq r$ and $p \notin\{i, j\}$. Let $c_{0}$ be the red-white coloring of $T$ obtained by recoloring $v_{i, i}$ and $v_{j, j}$ white and all other vertices of $T$ remain the same colors as in $c$. Since $d\left(v_{i, p}, w\right)=d\left(v_{j, p}, w\right)$ for every red vertex $w$ such that $w \notin\left\{v_{i, i}, v_{j, j}\right\}$, it follows that $\vec{d}_{c_{0}}(x)=\vec{d}_{c_{0}}(y)=\left(f_{1}, f_{2}, \ldots, f_{2 r}\right)$. Observe that $d\left(v_{i, p}, v_{i, i}\right)=i-p, d\left(v_{i, p}, v_{j, j}\right)=j+p, d\left(v_{j, p}, v_{i, i}\right)=i+p$, and $d\left(v_{j, p}, v_{j, j}\right)=j-p$. Since $i-p<\min \{i+p, j-p, j+p\}$, it follows that $a_{i-p}=f_{i-p}+1$ and $b_{i-p}=f_{i-p}$ and so $\vec{d}(x) \neq \vec{d}(y)$.

Subcase 2.2.2. $p \neq q$. First, suppose that $j \neq r$. Since (1) the last nonzero coordinate in $\vec{d}\left(v_{i, p}\right)$ is the $(p+r)$ th coordinate where $p+r=d\left(v_{i, p}, v_{r, r}\right)$ and the last nonzero coordinate in $\vec{d}\left(v_{j, q}\right)$ is the $(q+r)$ th coordinate where $q+r=d\left(v_{j, q}, v_{r, r}\right)$ and (2) $p \neq q$, it follows that either $a_{p+r} \neq b_{p+r}$ or $a_{q+r} \neq b_{q+r}$, implying that $\vec{d}(x) \neq \vec{d}(y)$.

Next, suppose that $j=r$. Thus, $x=v_{i, p}$ where $2 \leq i \leq r-1$ and $1 \leq p \leq i-1$ and $y=v_{r, q}$ where $1 \leq q \leq r-1$ and $p \neq q$. If $i-p \neq p+\ell$ for some $\ell \in[r]-\{i\}$, then $\left(1^{i-1}, 0,1^{r-i}\right)$ is a subsequence of $\vec{d}(x)$ and so there is no coordinate 2 in $\vec{d}(x)$. If $\vec{d}(y)=\vec{d}\left(v_{r, q}\right)$ contains 2 as a coordinate, then $\vec{d}(x) \neq \vec{d}(y)$ and so $\vec{d}(y)=\vec{d}\left(v_{r, q}\right)=\left(0^{r-q-1}, 1,0^{2 q-r}, 1^{r-1}, 0, \ldots, 0\right)$. Thus, $\vec{d}(x) \neq \vec{d}(y)$. Hence, we may assume that $i-p=p+\ell$ for some $\ell \in[r]-\{i\}$ and so $i=2 p+\ell$. This implies that there is exactly one coordinate of $\vec{d}(x)$ which is 2 , namely $a_{i-p}=2$. If $\vec{d}(y)$ has no coordinate 2 , then $\vec{d}(x) \neq \vec{d}(y)$. Hence, we assume that $\vec{d}(y)$ has 2 as a coordinate. This implies that $d\left(v_{r, q}, v_{r, r}\right)=r-q=q+t$ for some $t \in[r-1]$ and $b_{r-q}=2$ is the only coordinate 2 in $\vec{d}(y)$. Hence, $i-p=r-q$ or $r-i=q-p$ and so $q>p$. There are two possibilities here. If $i-p=r-q<p+1$, then the second nonzero coordinate in $\vec{d}(x)$ is $a_{p+1}$ while the the second nonzero coordinate in $\vec{d}(y)$ is $b_{q+1}$. If $i-p=r-q>p+1$, then the first nonzero coordinate in $\vec{d}(x)$ is $a_{p+1}$ while the the fist nonzero coordinate in $\vec{d}(y)$ is $b_{q+1}$. In either case, $\vec{d}(x) \neq \vec{d}(y)$.

Therefore, $c$ is an ID-coloring and so $\operatorname{ID}(T)=r$.

Several problems are suggested by the results presented here.

(1) For a given integer $r \geq 3$, what is the smallest order of a tree $T$ such that $\operatorname{ID}(T)=r$ ?

(2) For a given integer $r \geq 3$, what is the smallest order of a twin-free tree $T$ such that $\operatorname{ID}(T)=r$ ?

For (2), we have seen that this smallest order is no more than $1+\left(\begin{array}{c}r+2 \\ 2\end{array}\right)$.

\section{Acknowledgment}

We are grateful to Prof. Gary Chartrand for suggesting us concepts and kindly providing useful information on this topic.

\section{References}

[1] A. Ali, A survey of antiregular graphs, Contrib. Math. 1 (2020) 67-79.

[2] A. Ali, G. Chartrand, P. Zhang, Irregularity in Graphs, Springer, New York, 2021.

[3] G. G. Chappell, J. Gimbel, C. Hartman, Bounds on the metric and partition dimensions of a graph, Ars Combin. 88 (2008) $349-366$.

[4] G. Chartrand, L. Eroh, M. A. Johnson, O. R. Oellermann, Resolvability in graphs and the metric dimension of a graph, Discrete Appl. Math. 105 (2000) 99-113.

[5] G. Chartrand, Y. Kono, P. Zhang, Distance vertex identification in graphs, J. Interconnection Netw. 21 (2021) Art\# 2150005.

[6] G. Chartrand, E. Salehi, P. Zhang, The partition dimension of a graph, Aequationes Math. 59 (2000) 45-54.

[7] G. Chartrand, P. Zhang, The theory and applications of resolvability in graphs - a survey, Congr. Numer. 160 (2003) 47-68.

[8] F. Foucaud, G. B. Mertzios, R. Naserasr, A. Parreau, P. Valicov, Identification, location-domination and metric dimension on interval and permutation graphs. I. Bounds, Theoret. Comput. Sci. 68 (2017) 43-58.

[9] F. Foucaud, G. B. Mertzios, R. Naserasr, A. Parreau, P. Valicov, Identification, location-domination and metric dimension on interval and permutation graphs. II. Algorithms and complexity, Algorithmica 78 (2017) 914-944.

[10] F. Harary, R. A. Melter, On the metric dimension of a graph, Ars Combin. 2 (1976) 191-195.

[11] M. Hauptmann, R. Schmied, C. Viehmann, Approximation complexity of metric dimension problem, J. Discrete Algorithms 14 (2012) 214-222.

[12] C. Hernando, M. Mora, M. I. Pelayo, C. Seara, D. Wood, R. David, Extremal graph theory for metric dimension and diameter, Electron. J. Combin. 17 (2010) Art\# R30.

[13] M. A. Johnson, Structure-activity maps for visualizing the graph variables arising in drug design, J. Biopharm. Statist. 3 (1993) $203-236$.

[14] M. A. Johnson, Browsable structure-activity datasets, In: R. Carbó-Dorca, P. G. Mezey (Eds.), Advances in Molecular Similarity, Vol. 2, JAI Press Inc., Stamford, 1998, pp. 153-170.

[15] P. J. Slater, Leaves of trees, Congr. Numer. 14 (1975) 549-559.

[16] P. J. Slater, Dominating and reference sets in graphs, J. Math. Phys. Sci. 22 (1988) 445-455.

[17] P. Zhang, Color-Induced Graph Colorings, Springer, New York, 2015.

[18] P. Zhang, A Kaleidoscopic View of Graph Colorings, Springer, New York, 2016. 